УДК 65.011 .56

\title{
УПРАВЛЕНИЕ ПРЕДПРИЯТИЕМ НА ОСНОВЕ ИНТЕГРИРОВАННЫХ СРЕДСТВ ПОДДЕРЖКИ РАСПРЕДЕЛЁННЫХ БАЗ ДАННЫХ
}

\author{
(C) 2012 г. И. В. Баранова
}

\section{Московский государственный технологический университет «Станкин»}

Рассмотрены вопросы управления предприятием на основе использования интегрированных средств поддержки распределенных баз данных. Исследованы различные структуры информационных систем, в том числе рассмотрены преимущества и недостатки параллельной и распределенной архитектуры системы управления базами данных. Обоснована иелесообразность применения на промышленном предприятии при создании интегрированных средств поддержки распределенных баз данных поисковых машин.

Ключевые слова: система управления базами данных; поисковая машина; интегрированные средства поддержки распределенных баз данных; реляциионная модель.

The problems of the enterprise management, based on using of the integrated tools to support distributed databases are reviewed in the article. The different structures of information systems are also examined; the advantages and disadvantages of parallel and distributed system database management architecture are discussed. The utility of using the integrated tools to support distributed database search engines by the industrial plants is substantiated.

Key words: database management system; search engine; integrated support for distributed databases; object-oriented approach to the development of database management system; objectrelational database management system; parallel architecture of database management system; distributed architecture of database management systems; relational model.

Отличительными характеристиками современного машиностроительного производства являются высокий уровень технического и технологического оснащения (применение робототехники, высокоточного и высоконадежного станочного оборудования, в том числе и станков с ЧПУ), развитая система материально-технического обеспечения и высокий уровень квалификации персонала, участвующего в реализации бизнес-процессов на всех стадиях и этапах производственного цикла [1]. Поэтому для корректного и бесперебойного протекания всех стадий производства, начиная от выполнения НИОКР и заканчивая сбытом готовой продукции, необходимо наличие всеобъемлющей системы информационного обеспечения соответствующего уровня. Структура современного машиностроительного производства сложна и многогранна, характеризуется наличием разнородных информационных потоков. Все эти факторы, в свою очередь, обуславливают возникновение на уровне предприятия целого ряда проблем. Эти проблемы, например, могут быть связаны с несовпадением стандартов обрабатываемых данных, следствием чего являются трудности совместного отбора, анализа и использования этих данных [2;3].

Обозначенные нами проблемы могут быть решены различными способами, начиная от применения небольших «программшлюзов» и заканчивая созданием специализированного программно-аппаратного комплекса, адаптированного к условиям конкретного производственного процесса [4]. Наиболее целесообразно для решения этих проблем использовать информационные решения, основанные на применении систем управления базами данных (СУБД). Исходя 
из этого, необходимо рассмотреть концепцию оптимальной организации доступа к разнородным данным в условиях сложной гетерогенной информационной среды современного машиностроительного производства с применением поисковой машины [6].

Применяемые в настоящее время информационные системы имеют различную структуру. Причем конкретная реализация информационной системы, т. е. ее структура, во многом предопределяется сферой практического использования информационной системы. Для автоматизации производственных процессов в условиях тесной интеграции стадий и этапов этих процессов использование СУБД разного рода становится все более актуальным. Классификация СУБД может быть осуществлена по разным классификационным признакам.

Однако с точки зрения построения системы управления предприятием на основе интегрированных средств поддержки распределенных баз данных классификацию СУБД целесообразно проводить по принципу их работы. При построении этой классификационной схемы необходимо учитывать слабые и сильные стороны СУБД, а также мировые тенденции в развитии того или иного подхода к реализации СУБД.

На первом этапе развития систем коллективного доступа к данным интенсивно развивалось такое направление как разработка так называемых, локальных СУБД. Эти системы проектировались для одного или нескольких приложений и реализовывались на одиночных компьютерах, не связанных друг с другом единой системой обработки данных. Однако поскольку структура современного машиностроительного производства постоянно усложняется, становясь все более сложной иерархической системой, то актуальной становится задача создания распределенных систем СУБД. Подобные системы ориентированы на использование многоуровневых сетевых информационных платформ, имеющих общие принципы хранения, доступа и передачи данных и ориентированных на взаимодействие с глобальными сетевыми технологиями. Безусловно, некоторые существующие в настоящее время локальные СУБД имеют возможность интеграции в сетевые информационные системы, однако при разра- ботке новых СУБД в подавляющем большинстве случаев разработчики ориентируются на системы управления базами данных глобального уровня [5].

В настоящее время наибольшее распространение среди сложных СУБД получила концепция создания реляционных систем управления базами данных, т. е. баз данных, в которых данные находятся в иерархической структуре, т. е. подчинены строгой, связке (реляции). Кроме того, активно развиваются принципы и технологии объектно-ориентированного подхода к разработке СУБД. Наличие объектной ориентации определяется тремя основными свойствами.

Во-первых, механизмом наследования, предполагающим возможность синтезировать новые типы объектов на основе уже существующих с использованием (наследованием, линейным или множественным) их атрибутов и процедур.

Во-вторых, инкапсуляцией, в основе которой лежит принцип, заключающийся в построении оболочки вокруг некоторого набора данных и программного кода, обрабатывающего эти данные. Причем проявление этого свойства предполагает, что внешний объект может повлиять на инкапсулируемые данные (получить к ним доступ) только так, как позволяет объект, им владеющий.

В-третьих, полиморфизмом, т. е. возможностью применять методы обработки данных без учета реальных типов объектов, данные о которых подлежат обработке. Таким образом, полиморфизм характеризует свойство объектов некоторого класса-потомка замещать своими атрибутами и операциями атрибуты и операции класса-родителя, имеющими те же имена и параметры.

В качестве примера внедрения объектноориентированных принципов в обычную реляционную структуру СУБД можно привести линейку решений объектно-реляционной системы управления базами данных Oracle [8]. Этот программный продукт имеет в своем арсенале как инструментарий для построения реляционной модели СУБД, так и возможности внедрения объектной методологии, включая проектирование и практическую реализацию реляционной модели СУБД.

Во временном разрезе становление методологии проектирования СУБД совпало с 
развитием технологий распределенных вычислений и параллельной обработки. Причем в этой области были достигнуты значительные успехи. В результате соединения наиболее значимых достижений в развития этих двух направлений информатизации производственных процессов возникли системы управления распределенными базами данных и системы управления параллельными базами данных.

Благодаря интеграции рабочих станций в распределенную среду становится возможным более эффективное распределение функций в этой среде. В этом ситуации прикладные программы выполняются на рабочих станциях (серверах приложений), а базы данных обслуживаются выделенными компьютерами, называемыми серверами баз данных. Это послужило источником развития таких распределенных структур, в которых в роли узлов выступают не просто настольные (Desktop) компьютеры, а специализированные промышленные серверы. Распределенная база данных (Distributed Database - DDB) [6] представляет собой coвокупность множества взаимосвязанных баз данных, распределенных в многоуровневой компьютерной сети.

Система управления распределенной базой данных определяется как программная система, которая позволяет управлять базой данных таким образом, чтобы ее «распределенность» была прозрачна для пользователя. При этом распределенная база данных имеет ряд отличительных особенностей. Наиболее значимыми из них являются следующие особенности:

- наличие множества узлов приема запросов и множества узлов данных;

- слабосвязанный характер среды, означающий, что каждый узел имеет собственную операционную систему и функционирует независимо от других узлов;

- наличие механизмов распределения данных (горизонтальная и вертикальная фрагментация, их репликация или тиражирование);

- наличие иерархических отношений в распределенной базе данных (много клиентов - один сервер; много клиентов - много серверов; равный-к-равному).
Параллельные базы данных также имеют ряд отличительных черт. В качестве основных из этих черт можно назвать следующие признаки:

- проявление принципа «многопроцессорного компьютера», например, возможность определить параллельную СУБД как реализацию для многопроцессорного компьютера;

- наличие относительно жестких аппаратно-программных связей, например, возможность непосредственного переноса существующих СУБД на другую программную платформу с переработкой лишь интерфейса к операционной системе;

- наличие параллелизма, т. е. одновременное выполнение каких-либо манипуляций с базой данных;

- наличие возможности фрагментации функций, а также фрагментации данных.

Имея ряд различий, СУБД параллельной и распределенной архитектуры, тем не менее, во многом дополняют и обогащают друг друга. Можно выделить наиболее значимые идентифицирующие признаки параллельных и распределенных СУБД. Во-первых, распределенная/параллельная база данных - это именно база данных, а не «коллекция» файлов, индивидуально хранимых на разных узлах сети. В этом заключается ключевое различие между распределённой базой данных и распределенной файловой системой. Распределенные данные представляют собой распределенную базу данных, только если они связаны в соответствии с некоторым структурным формализмом (например, таким как реляционная модель), а для доступа к ним имеется единый и унифицированный высокоуровневый интерфейс.

Во-вторых, система обладает полной функциональностью СУБД. По своим возможностям эта функциональность не сводится ни к распределенным файловым системам, ни к системам обработки транзакций. Наряду с обработкой транзакций эти системы должны также обеспечивать реализацию функций запросов и структурной организации данных. Причем эти функции необязательно поддерживаются системой обработки транзакций. В-третьих, распределенная/параллельная база данных предполагает наличие распре- 
деления (включая фрагментацию и репликацию) данных по множеству узлов невидимых для пользователей.

В распределенных СУБД данные и приложения, которые осуществляют доступ к ним, могут быть локализованы на одном и том же узле. Благодаря этому свойству распределенных СУБД сокращается (а иногда полностью и исключается) потребность в удлиненном доступе к данным, характерная для систем телеобработки данных в режиме разделения времени. Поскольку на каждом узле распределенных СУБД выполняется меньше приложений и хранится меньшая порция базы данных, то в системе можно также сократить конкуренцию при доступе к данным и системным ресурсам.

Обеспечение высокой производительности является одной из важнейших целей, на достижение которой направлено использование технологий построения параллельных СУБД. Как правило, это обеспечивается за счет сочетания нескольких взаимодополняющих решений, например, таких как применение операционных систем, ориентированных на поддержку баз данных, создание в системе высокого уровня параллелизма, оптимизация системы, обеспечение баланса загрузки и т. д. Наличие операционной системы, «осведомленной» о специфических потребностях баз данных (например, потребности, касающейся управления буферами), упрощает реализацию функций баз данных нижнего уровня и способствует снижению стоимости выполнения этих функций. Так, например, в этом случае затраты на передачу сообщения могут быть значительно снижены (до нескольких сот инструкций) за счет применения специализированного коммуникационного протокола. Механизмы распределения способствуют повышению общей пропускной способности системы и снижению времени отклика для отдельных транзакций.

Технология построения распределенных и параллельных СУБД также обеспечивает повышение надежности, поскольку благодаря репликациям данных исключаются одиночные точки отката. Отказ одного узла или сбой на линии связи не приводит к выходу из строя всей системы. Даже если часть данных становится недоступной, при параллельной организации системы пользователи могут иметь доступ к остальной части информации. В среде параллельных и распределенных СУБД упрощается решение вопросов, связанных с возрастанием объема баз данных или потребностей обработки. При этом редко возникает необходимость в кардинальной перестройке системы. Расширение возможностей обычно достигается за счет добавления процессорных возможностей или памяти.

Принимая во внимание соотношение «цена/производительность» экономически целесообразно формировать систему из нескольких небольших компьютеров, а не реализовать ее на эквивалентной по мощности одной большой машине. Множество коммерческих распределенных СУБД функционируют на ЭВМ и рабочих станциях именно по причине более выгодного соотношения «цена/производительность». Технологии, основанные на применении рабочих станций, получили в настоящее время широкое распространение благодаря тому, что большинство коммерческих СУБД способны работать в рамках локальных сетей, где в основном используются рабочие станции. Развитие распределенных СУБД, предназначенных для глобальных сетей, может привести к повышению роли мейнфреймов. С другой стороны, можно предположить, что распределенные СУБД будущих поколений будут поддерживать иерархические сетевые структуры, состоящие из кластеров, в пределах которых компьютеры взаимодействуют на базе локальной сети, а сами кластеры соединяются между собой посредством высокоскоростных магистралей.

Исходя из характеристик параллельных и распределенных СУБД, можно выбрать наиболее распространенный промышленный вариант стандарта для СУБД. Этот вариант предполагает, что выбираются СУБД функционирующие в архитектуре клиент/сервер при двух и трёхзвенной реализации программных приложений. Возможно, в некоторых случаях аппаратная реализация таких СУБД будет иметь признаки параллелизма (наличие разделяемых дисков, совместно используемой памяти, многопроцессорность). Однако эти системы все равно следует относить к классу распределенных СУБД, а не к классу параллельных СУБД.

Вопросы организации быстрого и кор- 
ректного доступа к данным являются комплексными и на практике затрагивают как проблемы масштабирования, так и весь комплекс протоколов обеспечения надежности работы распределенной СУБД. Помимо этого, на качественные показатели доступа к данным влияет и такой, на первый взгляд второстепенный, фактор, как архитектурное решение рассматриваемой СУБД.

Но главной проблемой по-прежнему остается отсутствие эффективного и удобного для конечного пользователя инвариантного инструментария для легкого и наглядного поиска необходимой информации. Это особенно актуально для гетерогенных распределенных информационных сред, где данные не просто рассредоточены по различным узлам данных, но еще и хранятся на них в разных форматах. Весь спектр инструментальных средств, созданных в настоящее время, ограничивается предоставлением пользователю возможности самому явно выбрать стандарт той базы данных, к которой он хочет обратиться. Естественно, на практике пользователь не всегда знает о формате той или иной СУБД, а подчас и не имеет времени вникать в тонкости организации доступа к ней. Таким образом, один из главных вопросов современных СУБД является вопрос создания универсального инструмента для обеспечения быстрого, корректного и удобного доступа к базам данных различных по своей архитектуре и используемым форматам хранения данных.

В настоящее время существуют программные средства, позволяющие несколько упростить работу по созданию запросов к разнородным СУБД. Это достигается посредством применения языка SQL (Structured Query Language) [7; 8], а также путем масштабирования или репликации данных из одной базы данных в другую. Практически каждая фирма-разработчик реляционных СУБД в комплект ее поставки включает подобный инструмент. Наиболее типичными и широко распространенными представителями этого класса программного обеспечения является программное обеспечение промежуточного слоя, например, Open Database Connectivity (ODBC) компании Microsoft [7], входящее в комплект поставки операционных систем семейства Windows, и поставляемое вместе другими программными продуктами, а так- же Java DataBase Connectivity (JDBC) [8] платформенно-независимый промышленный стандарт взаимодействия Java-приложений с различными СУБД, реализованный в виде пакета java.sql, входящего в состав Java SE. Платформенно-независимый промышленный стандарт взаимодействия JDBC основан на концепции драйверов, позволяющих получать соединение с базой данных по специально описанному URL. Драйверы могут загружаться динамически (во время работы программы). Загрузившись, драйвер сам регистрирует себя и вызывается автоматически, когда программа требует URL, содержащий протокол, за который драйвер отвечает.

B арсенале как ODBC, так и JDBC есть набор библиотек, организующих доступ к базам данных различных форматов. В набор функций ODBC и JDBC входят механизмы обработки SQL-запросов в диалоговом режиме, поддержки хранимых процедур, модифицируемые курсоры и некоторые другие важные функции управления данными.

Для нормального масштабирования данных и их переноса из одной базы в другую, необходимо иметь инструмент для организации корректного доступа в них. Как и ODBC, так и JDBC имеют в своем активе драйверы для доступа к серверам таких баз данных, как Oracle, Microsoft SQL Server, Sybase SQL Server, Informix, MySQL, PostgreSQL, к таблицам локальных баз данных типа Paradox, dBase, Excel и др. При этом к основным минимальным функциональным возможностям программного обеспечения промежуточного слоя можно отнести:

- открытие и закрытие базы данных;

- поиск и инициализация значений свойств объектов;

- чтение и запись данных в объектах базы данных;

- создание таких объектов базы данных, как таблицы и индексы и т. д., а также работа с ними;

- создание псевдонимов баз данных (локальных серверов), их удаление и изменение;

- выполнение операций с базами данных типа копирования данных из одной базы в другую.

Обращение посредством ODBC или JDBC к базе данных происходит по уникальному псевдониму, который можно задать для 
этой базы в утилите конфигурирования. Подобная организация доступа намного упрощает работу с СУБД разных форматов, созданных на различных платформах. Работа с крупными информационными массивами на персональной ЭВМ в различных средах (клиент/сервер, Internet/intranet) связана с необходимостью решать целый ряд проблем. Если хранение информации осуществляется благодаря возможностям аппаратных средств и операционной системы, то поиск и манипулирование информации, а также включение в алгоритм и принятие решений целиком ложится на соответствующее программное обеспечение.

Под поисковой машиной понимается инвариантный по отношению к формату данных, хранимых в соответствующей базе, программный инструмент, позволяющий обеспечить поиск, обработку и удобное для пользователя представление искомой информации. При этом поисковая машина выполняет ряд функций. Наиболее значимыми из них являются следующие функции: прием запроса от пользователя; опрос возможных источников информации; сбор поступивших от потенциальных источников ответов; обработка полученных ответов; оформление отчета в формате, необходимом пользователю.

Принципиальное отличие концепции виртуальной поисковой машины от «программ-шлюзов» заключается в том, что поисковая машина сама распознает формат СУБД (будь то локальные таблицы, серверы удаленного доступа, XML- или HTML-страницы, используемые в Internet/intranet-сети) и проводит их обработку в соответствии с требованиями, предъявляемыми со стороны пользователя. Таким образом, отпадает необходимость «ручной настройки» приложения-клиента под конкретную СУБД, и, следовательно, для конечного пользователя процесс настройки становится «прозрачным», а получение результата запроса происходит за меньшее время. Структурная схема работы поисковой машины показана на рис. 1.

Поисковая машина интегрируется в схему «СУБД - приложение-клиент» в качестве программного обеспечения промежуточного слоя (рис. 2) и может располагаться как на отдельной рабочей станции значения, так и на главном (ведущем, головном) сервере.
При этом важно создать поисковой машине по возможности лучшие условия для скорейшего отбора необходимых данных в наибольшей зоне поиска.

В качестве практического использования поисковой машины можно привести следующий пример. Пользователю, например, технологу, работающему в технологическом бюро цеха, для запуска изделия в опытное производство требуется набор режущего инструмента с определёнными геометрическим характеристиками и прочностными параметрами. Пользователь формирует запрос к распределённой базе данных, используя, например, визуальный построитель запросов (Visual Query Builder) и отправляет его для выполнения.

Поисковая машина на основе файла конфигурации, содержащего реквизиты, перечень доступных локальных ЭВМ и узлов в сети поиска, а также поисковый алгоритм на основе приоритетов, осуществляет извлечение информации с её последующим накоплением в промежуточном файле, безусловно, если результат поиска не является пустым множеством значений.

Далее согласно требованиям, предъявляемым со стороны пользователя, формируется выходной документ - ведомость оснастки или карта заказа оснастки (в случае, если информация не найдена), отображаемый на экране дисплея ЭВМ. В случае необходимости твёрдую копию документа можно получить на устройстве печати и сохранить на жёстком диске локальной ЭВМ для дальнейшего использования в промышленном производстве.

Таким образом, в результате поисковых операций просмотрена и упорядочена, в соответствии с требованиями, информация, находящаяся на локальных ЭВМ, серверах технологического назначения, Internet/intranet серверах - в случае виртуальной промышленной производственной корпорации.

Изложенный подход, заключающийся в применении универсального программного обеспечения промежуточного слоя (ODBC и/или JDBC), а также в построении и реализации программного обеспечения поисковой машины может принести предприятию существенную выгоду за счёт унификации языка и программных средств построения запросов, 


\section{Приложение - клиент}

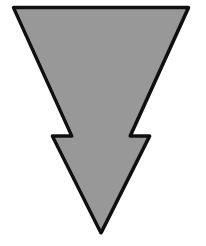

Запрос

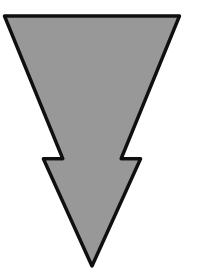

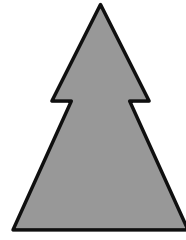

Отчет

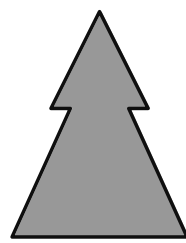

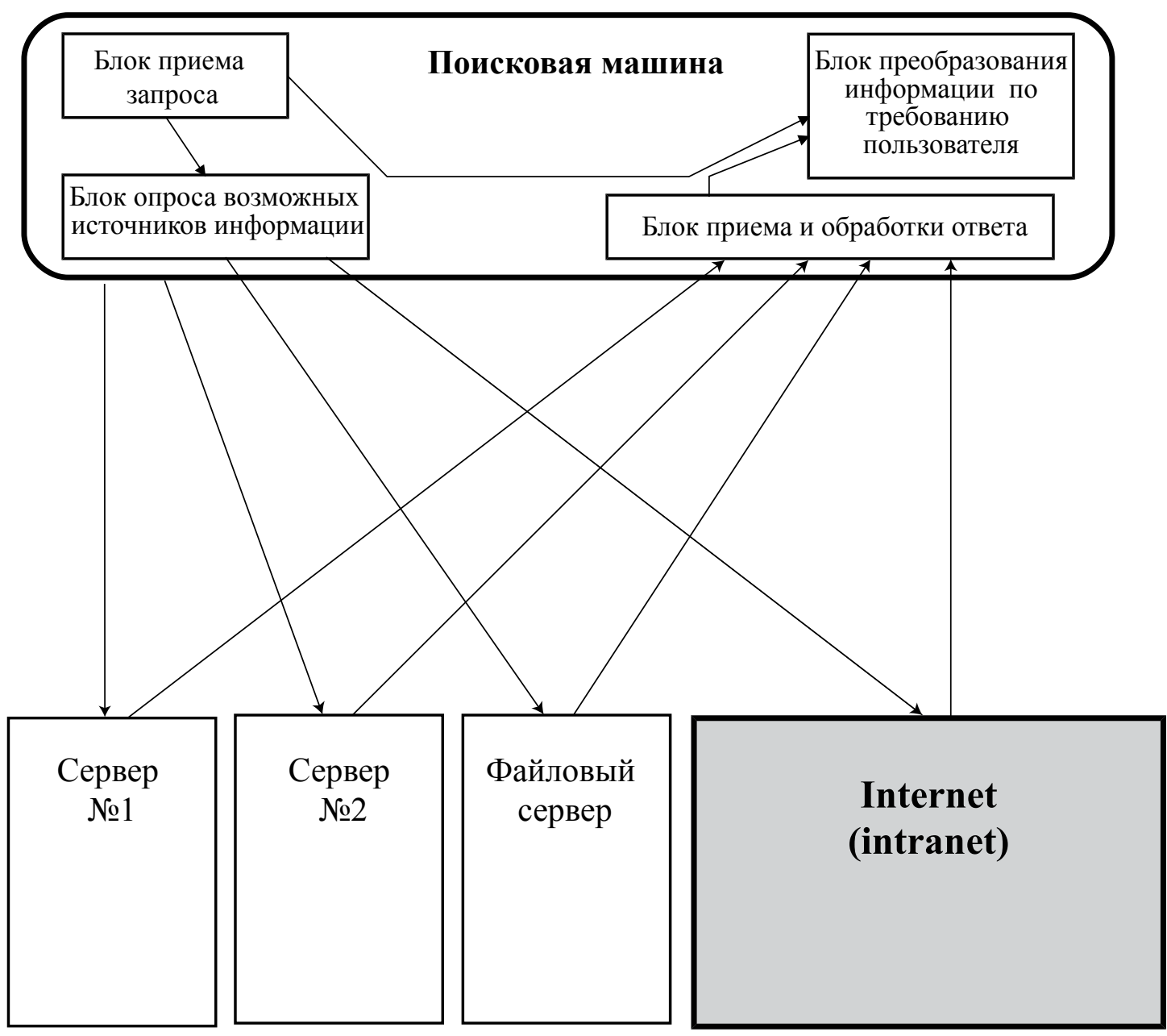

Рис. 1. Структурная схема работы поисковой машины 


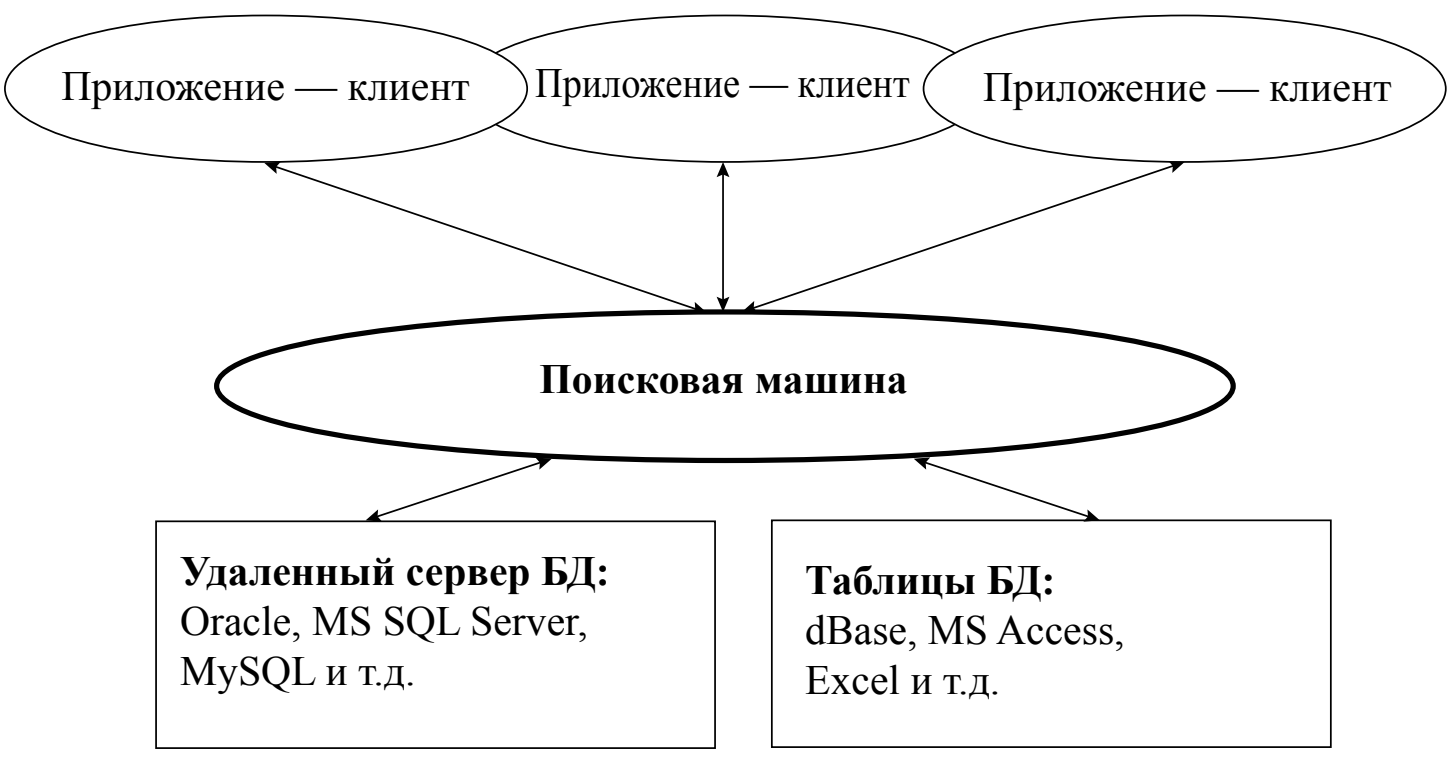

Рис. 2. Схема интеграции поисковой машины

практически независящих от вида и типа информации, хранимой в различных распределённых базах данных.

\section{Литература}

1. Батова М. М. Ковшов Е. Е. Смирнов $O$. С. Разработка информационных систем инновационного промышленного предприятия на основе унифицированного модульного подхода. // Инновации. - 2011. №5 (151). - С. 102-106.

2. Батова М. М., Ковшов Е. Е., Митропольский Н. Н. Комплексный подход в интеллектуальном анализе данных прикладной информационной системы. // Вестник Университета. Развитие отраслевого и регионального управления. - 2011. - №9. - С. 86-89.

3. Костров И. А. Ковшов Е. Е. Сервисноориентированная архитектура приложений как средство организации распределенных систем в среде слабоструктурированных данных. // Вестник МГТУ «Станкин». 2012. - №3 (22). - C. 140-145.

4. Борисенко Е. В., Ковшов Е. Е. Применение инструментальных средств обработки корпоративной информации на основе программно-аппаратных технологий. // Вестник МГТУ «Станкин». — 2010. — №3. - С. 123129.

5. Ковшов Е. Е., Смирнов О. С. Методы оценки и повышения надёжности информационных сред промышленного предприятия. // Динамика сложных систем - XXI век. 2012. — №2. - С. 112-116.

6. Стамировски Е. Т., Шемелин В. К., Ковшов Е. Е., Косов М. Г. Автоматизация доступа к информационным ресурсам распределенных баз данных современного производства. // Мехатроника, автоматизация, управление. - 2004. - №1. - С. 10-18.

7. ODBC - Open Database Connectivity Overview [Электронный pecypc] / Microsoft Corporation. - Режим доступа: http://support. microsoft.com/kb/110093, свободный. - Загл. с экрана.

8. Java SE Technologies - Database [Электронный pecypc] / Oracle Technological Network. - Режим доступа: http://www. oracle.com/technetwork/java/javase/jdbc/index. html, свободный. - Загл. с экрана. 


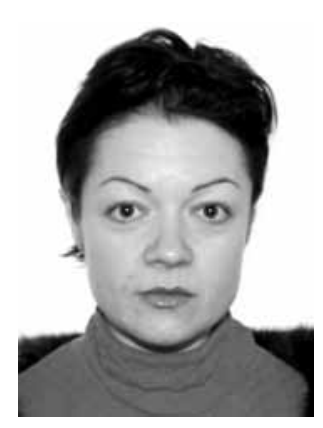

Ирина Вячеславовна Баранова - аспирантка кафедры «Управление и информатика в технических системах» Московского государственного технологического университета «Станкин».

Область научных интересов - использование математических и инструментальных методов для управления инновационными процессами в сложных экономических системах.

Автор четырех научных работ по проблемам разработки инструментальных средств и формирования экономико-математических моделей управления инновационными процессами промышленного предприятия.

Irina Vyacheslavovna Baranova - postgraduate student at the Management and Information Science in Engineering Systems department of the Moscow State Technological University «Stankin».

Research interests - using the mathematical methods and tools for management of the innovation processes in complex economic systems.

Author published four scientific papers on the development of tools and formation of economic and mathematical models for managing the innovative processes at the industrial enterprise.

127562, Москва, ул. Хачатуряна, д. 12, корп. 1, кв. 145

12 Khachaturiana st., bld. 1, app. 145, 127562, Moscow, Russia

Тел.: +7 (495) 755-62-41; +7 (903) 755-62-41; e-mail: yar.baranow@gmail.com 